\title{
Lumbopelvic Sagittal Alignment and Foraminal Height from Single Interbody Cage in L5-S1 Segment: Comparison between Anterior Cage for OLIF (Oblique Lumbar Interbody Fusion) and Curvilinear Cage for TLIF (Transforaminal Interbody Fusion)
}

\author{
Seong-Gon Kim, Jong-Tae Kim, Myung-Hoon Shin, Du-Yong Choi \\ Departments of Neurosurgery, Incheon St Mary's Hospital, College of Medicine, The Catholic University, Incheon, Korea
}

Corresponding Author: Jong-Tae Kim, $\mathrm{MD}, \mathrm{PhD}$

Department of Neurosurgery, Incheon St Mary's Hospital, College of Medicine, The Catholic University of Korea, 56 Dongsu-ro, Bupyeong-gu, Incheon 21431, Korea

Tel: $+82-32-280-5973$

Fax: +82-32-280-5991

E-mail: kjtnsa@naver.com

Received: February 6, 2019 Revised: April 11, 2019 Accepted: May 28, 2019

\begin{abstract}
Objective: To evaluate a radiological difference between the oblique lumbar interbody fusion (OLIF) L5-S1 and transforaminal lumbar interbody fusion (TLIF) L5-S1 procedure. Methods: 16 patients who underwent single-level OLIF L5-S1 and 32 patients of TLIF L5-S1 were retrospectively reviewed. Assessment of the lumbopelvic parameters and the height of disc space and L5-S1 neural foramen was performed before surgery as well as a month after surgery. Results: Preoperatively, OLIF L5-S1 group and TLIF group showed similar lumbar lordosis (LL), segmental lordosis (SL), pelvic incidence (PI), pelvic tilt (PT) and sacral slope (SS); $(p=0.08$, $0.48,0.67,0.19$ and 0.12 , respectively). Postoperatively those parameters did not show statistically significant differences between two groups $(p=0.17,0.32,0.53,0.16$ and 0.19 , respectively). In terms of disc and foraminal height, the OLIF L5-S1 group showed significant greater increase of posterior disc height and foraminal height ( $p=0.01$ and 0.00 ). There was no significant differences between the two groups in mean VAS and ODI score over the followup period. Conclusion: This study demonstrates that both OLIF L5-S1 group and TLIF L5-S1 group had similar ability of achieving lumbopelvic alignment however, increase of foraminal height was greater in patients with OLIF L5-S1 group.
\end{abstract}

Key Words: Oblique lumbar interbody fusion L5-S1, Transforaminal interbody fusion, Lumbopelvic parameters, Disc height, Foraminal height

\section{INTRODUCTION}

Oblique lumbar interbody fusion (OLIF) is a newly developed lumbar fusion technique that provides solution to disadvantages of direct lateral lumbar interbody fusion (DLIF). By using an oblique corridor bound by the psoas muscle or common iliac vein medially, large sized intervertebral cages can be lodged while mitigating the risk of injury to lumbar plexus. It has been believed that large sized cages are thought to be effective in restoring sagittal and coronal balance of patients with various spinal diseases ${ }^{3,6)}$. Transforaminal lumbar interbody fusion (TLIF) is a wellknown technique for achieving arthrodesis for various spinal diseases. The interbody cage used in TLIF not only provides anterior column support, restoration of disc height and nerve root decompression but also can act as fulcrum with resultant compression of the pedicel screw to lead to lordosis and improve- ment of lumbopelvic sagittal alignment ${ }^{4)}$.

Although radiological outcomes and complications of various lumbar interbody fusion have been investigated in many studies, OLIF L5-S1 technique for the degenerative spinal disease of elderly patients has been rarely reported. Furthermore, the comparison of radiological outcomes with TLIF L5-S1 has not been reported as far as the authors have known.

Therefore, the purpose of this study was to determine the changes of lumbopelvic parameters, disc height and foraminal height following OLIF L5-S1 and compare the differences of results with those of patients with TLIF L5-S1.

\section{Materials and Methods}

\section{Patients Selection}

We retrospectively reviewed the medical records of conse- 
cutive patients who underwent single level fusion surgery including OLIF L5-S1 and TLIF L5-S1 for lumbar degenerative disorders at a single institute from January 2016 to December 2019. Institutional review board approval was given before the initiation of medical record review for this study. Patients were required for preoperative and postoperative one month of radiological follow-up. The exclusion criteria were: 1) trauma, infection or malignancy; 2) in case of previous fusion surgery; 3) other kinds of surgery such as posterior lumbar interbody fusion or cases with cortical screws.

\section{Radiographic Assessment}

Plain radiographs of the lumbar spine were generally obtained preoperatively and a month after surgery. To investigate the changes of lumbopelvic sagittal alignment, we compared preoperative neutral lateral radiographs with those obtained during

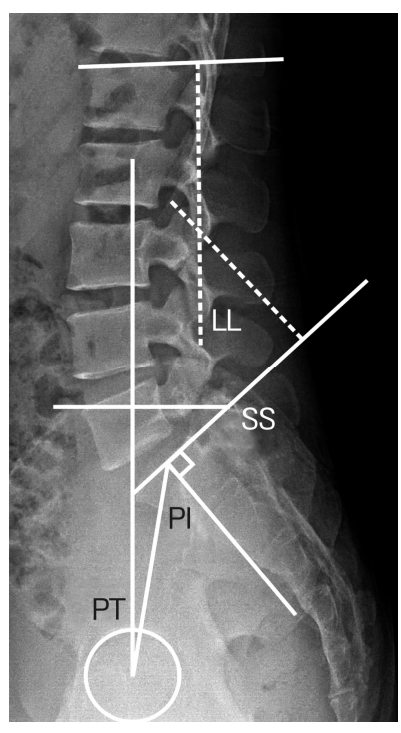

Fig. 1. Lumbopelvic parameters $\mathrm{LL}$; lumbar lordosis $\mathrm{Pl}$; pelvic incidence, PT; pelvic tilt, SS; sacral slope.
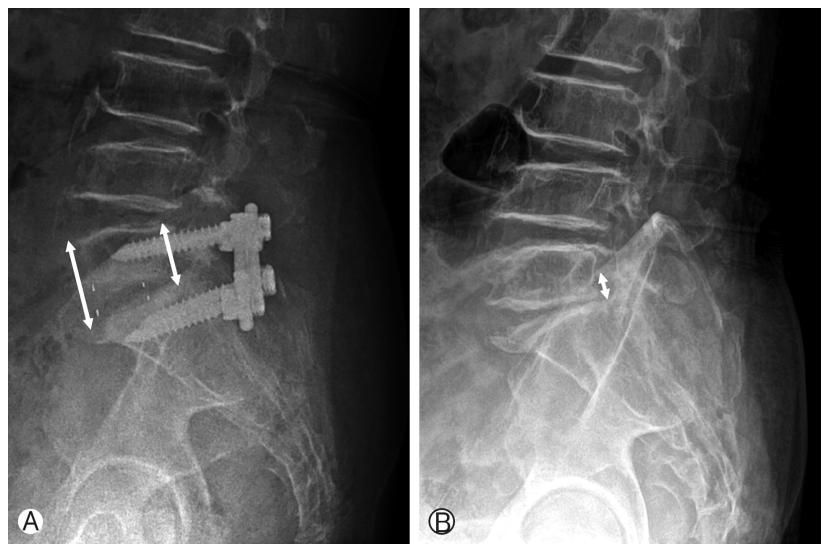

Fig. 2. Preoperative image of L5-S1 OLIF group depicting foraminal height (A). Postoperative image of L5-S1 OLIF group depicting anterior and posterior disc height (B). follow-up period. All radiographs were taken in a standing position and the lumbopelvic parameters measured. The segmental lordosis (SL) at the L5-S1 level was defined as the angle subtended by the superior endplate line of $L-5$ and endplate line of S-1. The angle of lumbar lordosis was measured from the inferior endplate of T12 to the superior endplate of S1 using the Cobb method. Pelvic incidence (PI) was defined as the angle between a line joining the center of sacral endplate to the bicoxo-femoral axis and a line perpendicular to the endplate. Pelvic tilt (PT) was defined as the angle formed by a line drawn from the midpoint of the sacral endplate to the center of the bicoxo-femoral axis and a vertical reference line. Sacral slope (SS) was defined as the angle formed by a line drawn along the endplate of the sacrum and a horizontal reference line (Fig. 1). The distance between inferior portion of L5 pedicle to upper portion of S1 pedicle was defined as a foraminal height and it was measured pre- and postoperatively (Fig. 2A). The mean disc height was regarded as a distance from $\mathrm{L} 5$ upper endplate to S1 upper end plate. It was measured at anterior and posterior corner of vertebral body to investigate the respective changes of anterior and posterior disc heights (Fig. 2B).

The radiographic measurements were taken by an independent fellowship-trained spine surgeon by using an automated program of the PACS computer software system and the mean value of two repeated measurement was used in analysis.

\section{Surgical Technique of OLIF L5-S1}

The patients were positioned in a true right lateral decubitus position with upper hip extended. After making the incision approximately 1-2 finger breadths (depending on patient size) from the anterior superior iliac spine (ASIS) and pelvis, the external oblique, internal oblique and transverse abdominal muscles were then dissected along the direction of their fibers. The retroperitoneal space was accessed by blunt dissection, and the peritoneal contents was retracted anteriorly. Once the common iliac artery and vein were identified, the retractor blade should be placed under the iliac vein after the adventitial layer has been released. Following exposure of L5-S1 disc space, annulotomy was done over the disc and, the disc material including the cartilaginous endplate was excised sequentially. An appropriate-sized spacer (Perimeter ${ }^{\mathbb{R}}$ cage; Medtronic Sofamor Danek, Memphis, TN) was filled with synthetic bone substitute and was inserted into the disc space. Pedicle screw fixation is performed by the conventional method.

\section{Surgical Technique of TLIF L5-S1}

The patients were placed on the Wilson frame in the prone position. Under fluoroscopic guidance, the proper level was marked and a standard midline incision was made. A subperiosteal dissection was followed to exposure the transverse process and pars interarticularis at the cephalad and caudal level. Initially with a rongeur and then with a high-speed drill, the medial aspect of the facet joints was excised and the lamina was thin- 
ned. After pedicle to pedicle decompression with removal of lateral part of facet joint, complete bilateral facetectomies were achieved. Ligamentum flavum on the symptomatic side was removed and then the ipsilateral exiting and traversing nerve root were released. Once the disc space was exposed clearly, the posterior annulotomy with a box cut was done and radical discectomy was performed using a combination of serial dilator and pituitary rongeurs. The disc and cartilage were removed carefully not to violate the subchondral bone and at this point, angled curette was used to remove the cartilaginous endplate from the far lateral side. A trial spacer was then used to estimate the appropriate size of cage and with protection of the thecal sac and root by retractor, interbody cage (curvilinear shaped Crescent $^{\circledR}$ cage; Medtronic Sofamor Danek, Memphis, TN) filled with graft material (cancellous autobone harvested from the laminal mixed with cancellous allograft) was inserted and additional bone chips were packed into the disc space.

\section{Clinical Assessment}

Clinical symptoms and functional outcomes were evaluated using the visual analog scale (VAS) and ODI pre- and postoperatively (in prior to release from hospital), at 6 months after surgery.

\section{Statistical Analysis}

The continuous variables were compared either using student's $t$-test after verification of normal distribution by the Kolmogorov-Smirnov test or using the non-parametric Mann-

Table 1. Basic demographics of OLIF L5-S1 and TLIF L5-S1 group

\begin{tabular}{lccc}
\hline \hline & OLIF L5-S1 & TLIF L5-S1 & $\mathrm{p}$ \\
\hline Number of patients & 16 & 32 & $\mathrm{NI}$ \\
Sex & & & \\
$\quad$ male & 6 & 15 & 0.75 \\
$\quad$ female & 10 & 17 & \\
Mean age (year) & $63.21 \pm 7.29$ & $64.25 \pm 5.46$ & 0.44 \\
BMD (T-score) & $-1.47 \pm 1.41$ & $-1.53 \pm 2.11$ & 0.81 \\
BMl (kg/m $\left.{ }^{2}\right)$ & $25.17 \pm 3.28$ & $24.38 \pm 5.14$ & 0.56 \\
\hline
\end{tabular}

$\mathrm{Nl}$ : not involved.
Whitney $U$ test. To compare the changes between before and after surgery, paired $t$-test or Wilcoxon signed rank test was used depending on result of normality test. P-value less than 0.05 was regarded to be statistically significant.

\section{RESULTS}

\section{Patient Demographics}

A total of 48 patients were enrolled for the analysis and they were divided into two groups according to operative procedure: OLIF L5-S1 or TLIF L5-S1 group. Sixteen patients (6 males, 10 females) were enrolled in the OLIF L5-S1 group and thirty-two patients (15 males, 17 females) were enrolled in TLIF L5-S1 group. Table 1 shows the comparison of patients' demographic data between two groups. Patients in the OLIF L5-S1 group were younger and heavier than those in the TLIF L5-S1 group however, they were statistically insignificant $(p=0.44$ and 0.56 , respectively) and the remaining $\mathrm{BMD}$, gender composition were similar as well ( $p=0.81$ and 0.75 , respectively).

\section{Radiographic outcomes}

Table 2 shows the comparison of lumbopelvic parameters between OLIF L5-S1 and TLIF L5-S1 group. In the setting of similar PI ( $p=0.67)$, OLIF L5-S1 groups had lower $L L$, lower SS and higher PT however, they were statistically insignificant $(p=$ $0.08,0.12$ and 0.19 , respectively). These similarities were persistent following the operation ( $\mathrm{p}=0.17,0.19$ and 0.16 , respectively).

Table 3 shows the changes of lumbopelvic parameters. In OLIF L5-S1 group, the mean value of LL and SS were increased following operation $\left(8.22 \pm 8.84^{\circ}\right.$ and $6.66 \pm 8.00^{\circ}$, respectively) with decrease of PT $\left(-5.33 \pm 6.70^{\circ}\right)$. Also in TLIF L5-S1 group, similar changes were observed (LL; 6.54 $\pm 9.08^{\circ}, \mathrm{SS} ; 6.13 \pm 8.88^{\circ}$ and PT; $-5.04 \pm 8.22^{\circ}$. In the comparison of changes of parameters between two groups, it was demonstrated that those changes were not statistically different between OLIF L5-S1 and TLIF L5-S1 group (LL; $p=0.10$, SS; $p=0.89$ and PT; $p=0.93$ ).

In the comparison of changes of disc and foraminal height (Table 4), the increase of anterior height was similar between two groups $(\mathrm{p}=0.19)$ however, OLIF L5-S1 group showed greater increase of posterior height compared with TLIF L5-S1 group $(3.05 \pm 3.35 \mathrm{~mm}$ vs $1.65 \pm 2.51 \mathrm{~mm}, \mathrm{p}=0.01)$. When it comes to

Table 2. Comparison of lumbopelvic parameters ( ${ }^{\circ}$ )

\begin{tabular}{|c|c|c|c|c|c|c|}
\hline & \multicolumn{3}{|c|}{ Preoperative } & \multicolumn{3}{|c|}{ Postoperative } \\
\hline & OLIF L5-S1 & TLIF L5-S1 & $p$ & OLIF L5-1 & TLIF L5-S1 & $p$ \\
\hline Lumbar lordosis & $30.22 \pm 13.21$ & $45.76 \pm 20.92$ & 0.08 & $38.44 \pm 9.67$ & $45.71 \pm 11.33$ & 0.17 \\
\hline Segmental lordosis & $14.00 \pm 6.36$ & $45.76 \pm 20.92$ & 0.48 & $19.55 \pm 5.52$ & $21.88 \pm 3.44$ & 0.32 \\
\hline Pelvic incidence & $52.60 \pm 7.71$ & $54.65 \pm 6.87$ & 0.67 & $51.33 \pm 7.98$ & $53.68 \pm 7.39$ & 0.53 \\
\hline Pelvic tilt & $21.77 \pm 6.13$ & $17.05 \pm 8.12$ & 0.19 & $16.44 \pm 6.10$ & $12.00 \pm 6.56$ & 0.16 \\
\hline Sacral slope & $27.66 \pm 9.57$ & $35.55 \pm 10.53$ & 0.12 & $34.33 \pm 10.78$ & $41.68 \pm 5.57$ & 0.19 \\
\hline
\end{tabular}


the change of foraminal height, $3.83 \pm 2.27 \mathrm{~mm}$ of increase was observed in OLIF L5-S1 group while $1.58 \pm 1.29 \mathrm{~mm}$ of increase was observed in TLIF L5-S1 group $(p=0.00)$.

\section{Clinical outcomes}

The two groups had comparable VAS scores preoperatively $(p=0.38$, Table 5). Postoperatively and at 6 months of follow-up, the two groups were found to have similar VAS outcome scores ( $p=0.55$ and 0.66 ). In terms of ODI score, there was no statistically significant difference between two group preoperatively and over the follow-period ( $p=0.48,0.78$ and 0.58 , respectively).

\section{DISCUSSION}

Lumbopelvic sagittal balance plays a essential role in maintaining sagittal posture. LL normally tried to position the center of body over the hip joint to maintain a balanced posture. However, in the situation of the maximal LL trying to keep the sagittal balance, the pelvis will naturally rotate backward. The backward rotation of pelvis can be defined as PT which reflecting the capacity of the pelvic posterior tilt. It is known that the larger the pelvic rotated backward, the larger the PT. Various studies have shown that both the OLIF and TLIF is effective in correcting the preoperative $L L$ and compensatory PT by restoring the intervertebral disc space ${ }^{1,7,8)}$. Xiao et al reported that both the increase of $\operatorname{LL}$ (from $38.52 \pm 8.02^{\circ}$ to $44.71 \pm 8.26^{\circ}$ ) and

Table 3. Changes of lumbopelvic parameters $\left(\Delta,{ }^{\circ}\right)$

\begin{tabular}{lrrc}
\hline \hline & OLIF L5-S1 & TLIF L5-S1 & $\mathrm{p}$ \\
\hline Lumbar lordosis & $8.22 \pm 8.84$ & $6.54 \pm 9.08$ & 0.10 \\
Segmental lordosis & $5.55 \pm 2.83$ & $6.06 \pm 1.96$ & 0.67 \\
Pelvic incidence & $0.44 \pm 1.50$ & $0.38 \pm 1.34$ & 0.37 \\
Pelvic tilt & $-5.33 \pm 6.70$ & $-5.04 \pm 8.22$ & 0.93 \\
Sacral slope & $6.66 \pm 8.00$ & $6.13 \pm 8.88$ & 0.89 \\
\hline
\end{tabular}

Table 4. Comparison of $(\Delta, \mathrm{mm})$

\begin{tabular}{lccc}
\hline \hline & OLIF L5-S1 & TLIF L5-S1 & $\mathrm{p}$ \\
\hline Anterior height & $6.75 \pm 2.59$ & $4.85 \pm 3.16$ & 0.19 \\
Posterior height & $3.05 \pm 3.35$ & $1.65 \pm 2.51$ & 0.01 \\
Foraminal height & $3.83 \pm 2.27$ & $1.58 \pm 1.29$ & 0.00 \\
\hline
\end{tabular}

Bolded values are statistically significant.
SS $\left(27.11 \pm 10.50^{\circ}\right.$ to $\left.34.09 \pm 11.09^{\circ}\right)$ following OLIF surgery. Ohtori et al. ${ }^{5}$ ) also found that OLIF significantly improved LL, PT and SS. In this study, the authors compared the restoration of lumbopelvic parameter between two kinds of minimally invasive lumbar arthrodesis techniques. With the assumption that the L5-S1 disc space has major portion of formatting lumbar lordosis, the authors hypothesized that the relatively large sized interbody cage of OLIF L5-S1 could excel the TLIF in terms of the restoration of lumbopelvic parameters. However, the changes of lumbopelvic parameters of both techniques were found to be similar each other. The similarity might be attributable to the fact that single level of intervertebral cages could not be enough to reveal the different capacity of restoration between two techniques.

In study of Champagne et al. ${ }^{6}$, it was found that OLIF was the only procedure inducing significant correction of lumbar lordosis ( $4.8^{\circ}$ correction, $\left.p=0.049\right)$ whereas TLIF did not affect it $\left(0.9^{\circ}\right.$ correction, $\left.p=0.65\right)$ and TLIF negatively corrected it $\left(-5.2^{\circ}\right.$ correction, $p=0.011)$. They believed easy access to anterior column and use of higher intervertebral cages of OLIF procedure could explain these results. They also reported the disc height elevation in regard to the approach used and explained that both the TLIF and OLIF managed to statistically significantly augment disc height with OLIF having the greatest impact (1.7 $\mathrm{mm}$, and $3.7 \mathrm{~mm}$ of correction for MIS TLIF and OLIF, with $p<0.001$ and $p<0.001$, respectively). Our results are similar. The length of elevated posterior disc height was greater in patients with OLIF L5-S1 group than that in patients with TLIF group whereas the anterior disc height was similar each other. We assume that this result is related with the position of the intervertebral cage. In case of TLIF group, curvilinear type cage was commonly used. Compared with straight type cage, curvilinear type cage is reported to be positioned anteriorly on the disc space ${ }^{2}$. Seemingly, the position of intervertebral cage seems to affect the elevation of intervertebral foramen. The change of intervertebral foramen in OLIF L5-S1 group is $3.83 \mathrm{~mm}$, which was statistically greater than that of the TLIF L5-S1 group $(1.5 \mathrm{~mm}, \mathrm{p}=0.003)$. We believe anteriorly positioned curvilinear cage could let the cantilever effect happened at the index level and it would be disadvantageous in making neural foramen widening. In contrast, relatively large sized of cages in OLIF L5-S1 could facilitate the increase of neural foraminal height.

There are some limitations to the current study that should be mentioned; the study was a retrospective design and the number of cohorts was relatively small to draw the conclusions.

Table 5. The comparisons of VAS and ODI (\%) between OLIF L5-S1 and TLIF L5-S1 group

\begin{tabular}{clccc}
\hline \hline & & Preoperatively & Postoperatively & Postop 6 months \\
\hline VAS & OLIF L5-S1 & $7.05 \pm 1.91$ & $2.77 \pm 2.34$ & $3.84 \pm 1.87$ \\
& TLIF L5-S1 & $6.54 \pm 2.32$ & $2.48 \pm 1.95$ & $3.52 \pm 1.56$ \\
& p & 0.38 & 0.55 & 0.66 \\
ODI & OLIF L5-S1 & $55.45 \pm 11.74$ & $21.86 \pm 8.64$ & $22.65 \pm 7.88$ \\
& TLIF L5-S1 & $53.21 \pm 12.55$ & $20.54 \pm 9.52$ & $21.33 \pm 6.97$ \\
& p & 0.48 & 0.78 & 0.58 \\
\hline
\end{tabular}


The follow-up period was relatively short, and it lacks timeline comparison between two groups. Lastly, radiological measurements of disc and foraminal heights were performed from simple lateral images and it might contain a radiological error. Prospective study with comparison between preoperative and follow-up CT scan images should be done in subsequent study.

\section{CONCLUSION}

In this retrospective study, we directly compared the two different types of minimal invasive lumbar arthrodesis technique OLIF L5-S1 and TLIF L5-S1. Despite similar lumbopelvic parameters, the posterior disc height and foraminal height change of OLIF L5-S1 group were better than those of the TLIF group. Future studies with a longer follow-up will be critical for clarifying clinical benefits of these findings.

\section{ACKNOWLEDGEMENTS}

The authors declare that they have no financial or other conflicts of interest in relation to this research and its publication.

\section{REFERENCES}

1. Champagne PO, Walsh C, Diabira J, Plante ME, Wang Z, Boubez G, et al: Sagittal balance correction following lumbar interbody fusion: A comparison of the three approaches. Asian Spine J 13: 450-458, 2019

2. Kim JT, Shin MH, Lee HJ, Choi DY: Restoration of lumbopelvic sagittal alignment and its maintenance following transforaminal lumbar interbody fusion (TLIF): Comparison between straight type versus curvilinear type cage. Eur Spine J 24:2588-2596, 2015

3. Kim KT, Jo DJ, Lee SH, Seo EM: Oblique retroperitoneal approach for lumbar interbody fusion from L1 to S1 in adult spinal deformity. Neurosurg Rev 41:355-363, 2018

4. Lazennec JY, Ramare S, Arafati N, Laudet CG, Gorin M, Roger $\mathrm{B}$, et al: Sagittal alignment in lumbosacral fusion: relations between radiological parameters and pain. Eur Spine J 9:47-55, 2000

5. Ohtori S, Mannoji C, Orita S, Yamauchi K, Eguchi Y, Ochiai $\mathrm{N}$, et a.: Mini-open anterior retroperitoneal lumbar interbody fusion: Oblique lateral interbody fusion for degenerated lumbar spinal kyphoscoliosis. Asian Spine J 9:565-572, 2015

6. Sharma AK, Kepler CK, Girardi FP, Cammisa FP, Huang RC, Sama AA: Lateral lumbar interbody fusion: clinical and radiographic outcomes at 1 year: a preliminary report. J Spinal Disord Tech 24:242-250, 2011

7. Sheng SR, Geng YB, Zhou KL, Wu AM, Wang XY, Ni WF: Minimally invasive surgery for degenerative spondylolisthesis: transforaminal or oblique lumbar interbody fusion. J Comp Eff Res 9:45-51, 2020

8. Xiao L, Zhao Q, Sun X, Liu C, Zhang Y, Xu H: Relationship between alterations of spinal/pelvic sagittal parameters and clinical outcomes after oblique lumbar interbody fusion. World Neurosurg 133:e156-e164, 2020 